\title{
Intrinsic linking and knotting are arbitrarily complex
}

\author{
by \\ Erica Flapan (Claremont, CA), Blake Mellor (Los Angeles, CA) \\ and Ramin Naimi (Los Angeles, CA)
}

\begin{abstract}
We show that, given any $n$ and $\alpha$, any embedding of any sufficiently large complete graph in $\mathbb{R}^{3}$ contains an oriented link with components $Q_{1}, \ldots, Q_{n}$ such that for every $i \neq j,\left|\operatorname{lk}\left(Q_{i}, Q_{j}\right)\right| \geq \alpha$ and $\left|a_{2}\left(Q_{i}\right)\right| \geq \alpha$, where $a_{2}\left(Q_{i}\right)$ denotes the second coefficient of the Conway polynomial of $Q_{i}$.
\end{abstract}

1. Introduction. The study of embeddings of graphs in $\mathbb{R}^{3}$ is a natural extension of knot theory. However, in contrast with knots, whose properties depend only on their extrinsic topology, there is a rich interplay between the intrinsic structure of a graph and the extrinsic topology of all embeddings of the graph in $\mathbb{R}^{3}$. Conway and Gordon [1] obtained groundbreaking results of this nature by showing that every embedding of the complete graph $K_{6}$ in $\mathbb{R}^{3}$ contains a non-trivial link and every embedding of $K_{7}$ in $\mathbb{R}^{3}$ contains a non-trivial knot. Because this type of linking and knotting is intrinsic to the graph itself rather than depending on the particular embedding of the graph in $\mathbb{R}^{3}, K_{6}$ is said to be intrinsically linked and $K_{7}$ is said to be intrinsically knotted. On the other hand, Conway and Gordon [1] illustrated an embedding of $K_{6}$ such that the only non-trivial link $L_{1} \cup L_{2}$ contained in $K_{6}$ is the Hopf link (which has $\left|\operatorname{lk}\left(L_{1}, L_{2}\right)\right|=1$ ); and they illustrated an embedding of $K_{7}$ such that the only non-trivial knot $Q$ contained in $K_{7}$ is the trefoil knot (which has $\left|a_{2}(Q)\right|=1$, where $a_{2}(Q)$ denotes the second coefficient of the Conway polynomial of $Q$ ). In this sense, we see that $K_{6}$ exhibits the simplest type of intrinsic linking and $K_{7}$ exhibits the simplest type of intrinsic knotting.

More recently, it has been shown that for sufficiently large values of $r$, the complete graph $K_{r}$ exhibits more complex types of intrinsic linking and knotting. In particular, Flapan [2] showed that for every $\lambda \in \mathbb{N}$, there is a

2000 Mathematics Subject Classification: 57M25, 57M15, 05C10.

Key words and phrases: intrinsically linked graphs, intrinsically knotted graphs. 
complete graph $K_{r}$ such that every embedding of $K_{r}$ in $\mathbb{R}^{3}$ contains both a 2-component oriented link $L$ whose linking number is at least $\lambda$ and a knot $Q$ with $\left|a_{2}(Q)\right| \geq \lambda$ (though $L$ and $Q$ have no particular relationship). Fleming [4] showed that for any $n \in \mathbb{N}$, there is a graph $G$ such that every embedding of $G$ in $\mathbb{R}^{3}$ contains a non-split link of $n+1$ components where $n$ of the components are non-trivial knots. In the current paper, we show that for sufficiently large complete graphs, intrinsic linking with knotted components is arbitrarily complex both in terms of linking number and in terms of the knotting of every component. In particular, our main result is the following.

TheOREM 2. For all $n, \alpha \in \mathbb{N}$, there is a complete graph $K_{r}$ such that every embedding of $K_{r}$ in $\mathbb{R}^{3}$ contains an oriented link with components $Q_{1}, \ldots, Q_{n}$ such that for any $i \neq j,\left|\operatorname{lk}\left(Q_{i}, Q_{j}\right)\right| \geq \alpha$ and $\left|a_{2}\left(Q_{i}\right)\right| \geq \alpha$.

If linking is measured with linking number and knotting is measured with $a_{2}$, then this is the strongest result one could hope for about the complexity of simultaneous intrinsic knotting and linking. Furthermore, observe that for a given $c \in \mathbb{N}$, there are only finitely many knots whose minimal crossing number is less than or equal to $c$. If we pick $\lambda$ larger than the $\left|a_{2}\right|$ of all of the knots with minimal crossing number less than or equal to $c$, then the knots $Q_{1}, \ldots, Q_{n}$ given by Theorem 2 will each have minimal crossing number greater than $c$. It follows that the complexity of intrinsic knotting as measured by the crossing number can also be made arbitrarily large.

In order to prove our main result, we first prove in Section 2 that intrinsic linking is arbitrarily complex in the sense of the structure of a link. In particular, we prove the following.

TheOREM 1. For all $n, \lambda \in \mathbb{N}$, there is a complete graph $K_{r}$ such that every embedding of $K_{r}$ in $\mathbb{R}^{3}$ contains an oriented link with components $Q_{1}, \ldots, Q_{n}$ such that for any $i \neq j,\left|\operatorname{kk}\left(Q_{i}, Q_{j}\right)\right| \geq \lambda$.

The idea of the proof of Theorem 2 is then as follows. Taniyama and Yasuhara [9] have shown that for any embedding of the pseudo-graph $D_{4}$ (see Figure 5), the product of the linking numbers of the two opposite pairs of cycles, $C_{i}$ and $C_{j}$, is related to the sum of the $a_{2}$ 's of all of the Hamiltonian cycles $Q$ according to the formula

$$
\sum_{Q \in S}\left|a_{2}(Q)\right| \geq\left|\operatorname{lk}\left(C_{1}, C_{3}\right) \operatorname{lk}\left(C_{2}, C_{4}\right)\right| .
$$

We use Theorem 1 to find a complete graph $K_{r}$ so that every embedding of $K_{r}$ contains a link with a "large enough" number of components all pairwise linked with linking number at least $\lambda$. We then use a recursive argument to successively exchange opposite pairs of linked cycles in a $D_{4}$ (which is a minor of $K_{r}$ ) for a knotted Hamiltonian cycle in $D_{4}$ which is linked with all 
of the previous knotted cycles in the construction. We do this in such a way that for all of the knotted cycles $Q_{i}$ and $Q_{j}$ we have $\left|\operatorname{lk}\left(Q_{i}, Q_{j}\right)\right| \geq \lambda \geq \alpha$ and $\left|a_{2}\left(Q_{i}\right)\right| \geq \lambda^{2} / 16 \geq \alpha$.

We wish to thank Alan Tarr for suggesting that we represent the structure of a link with a linking pattern, for reading a preliminary draft of this paper, and for suggesting that we prove the current version of Theorem 1. We also want to thank Kouki Taniyama for asking us whether the conclusion of Theorem 1 could be strengthened by removing the absolute value on the linking number. The current version of Proposition 2, together with Corollary 1, and Proposition 3 grew out of our response to Taniyama's question.

2. Intrinsic linking. We will use a weighted graph to describe the structure of a link as follows.

Definition 1. Given an oriented link $L$ with components $L_{1}, \ldots, L_{n}$, the linking pattern of $L$ is the graph with vertices $v_{1}, \ldots, v_{n}$ such that there is an edge between $v_{i}$ and $v_{j}$ if and only if $\operatorname{lk}\left(L_{i}, L_{j}\right) \neq 0$. The weighted linking pattern of $L$ is the linking pattern with a weight assigned to each edge $\left\{v_{i}, v_{j}\right\}$ representing the value of $\left|\operatorname{lk}\left(L_{i}, L_{j}\right)\right|$.

For example, the linking pattern of a Hopf link is a single edge. As another example, consider a keyring link, that is, a link consisting of a ring, $J$, and keys, $L_{1}, \ldots, L_{n}$, such that $\operatorname{lk}\left(J, L_{i}\right) \neq 0$ and $\operatorname{lk}\left(L_{i}, L_{j}\right)=0$ for all $i \neq j$. The linking pattern of a keyring link is an $n$-star (i.e., a graph consisting of $n$ vertices all connected to a single additional vertex).

Many results about intrinsic linking use the mod 2 linking number,

$$
\omega(J, L)=\operatorname{lk}(J, L) \bmod 2,
$$

as a simpler measure of linking than the ordinary linking number. Thus we will also use the following definition.

Definition 2. Given a link $L$ with components $L_{1}, \ldots, L_{n}$, the $\bmod 2$ linking pattern of $L$ is the graph with vertices $v_{1}, \ldots, v_{n}$ such that there is an edge between $v_{i}$ and $v_{j}$ if and only if $\omega\left(L_{i}, L_{j}\right)=1$.

Using this terminology, Conway and Gordon [1] and Sachs [7]-[8] showed that every embedding of $K_{6}$ in $\mathbb{R}^{3}$ contains a link whose mod 2 linking pattern is a single edge. Fleming and Diesl [5] showed that there is a graph $G$ such that every embedding of $G$ in $\mathbb{R}^{3}$ contains a link $J \cup L_{1} \cup \cdots \cup L_{n}$ where $\omega\left(J, L_{i}\right)=1$ for each $i$. Thus every embedding of $G$ in $\mathbb{R}^{3}$ contains a link whose mod 2 linking pattern contains an $n$-star, possibly with additional edges. We call such a link a generalized keyring link, since some of the $L_{i}$ 's may be linked with one another. 
Prior to this paper, a chain of $n$ edges and a circle of $n$ edges [3], and an $n$-star [5] were the only linking patterns $\Gamma$ which were known to have the property that for some graph $G$ every embedding of $G$ in $\mathbb{R}^{3}$ contains a link whose linking pattern contains $\Gamma$. We prove in Theorem 1 that for every complete graph $K_{n}$, there is a graph $G$ such that every embedding of $G$ in $\mathbb{R}^{3}$ contains a link whose linking pattern is $K_{n}$. It follows that for any linking pattern $\Gamma$, there is a graph $G$ such that every embedding of $G$ in $\mathbb{R}^{3}$ contains a link whose linking pattern contains $\Gamma$. In particular, using the language of linking patterns we prove the following restatement of Theorem 1.

Theorem 1. Let $\lambda \in \mathbb{N}$. For every $n \in \mathbb{N}$, there is a graph $G$ such that every embedding of $G$ in $\mathbb{R}^{3}$ contains a link whose linking pattern is $K_{n}$ with every weight at least $\lambda$.

This theorem implies that given any $n, \lambda \in \mathbb{N}$, there exists a graph $G$ which has the property that every embedding of $G$ contains an $n$-component link all of whose components are pairwise linked with the absolute value of their linking number at least $\lambda$ (this was our statement of Theorem 1 in the introduction). Furthermore, in Proposition 2 we show that complete graphs are the only linking patterns that have the property described by Theorem 1 . In other words, complete graphs can be said to be the only intrinsic linking patterns.

Before we prove Theorem 1, we will show in Proposition 1 that for every $n$, there is a graph such that every embedding of the graph contains a link whose linking pattern contains the complete bipartite graph $K_{n, n}$. We will use this result to prove Theorem 1. In fact, in Proposition 1, we prove the stronger result that we can ensure that all of the weights are odd. It is an open question whether this stronger formulation can be extended to the linking pattern $K_{n}$.

In order to prove Proposition 1, we need the following lemma which allows us to combine many pairs of linked cycles into a single cycle that links some proportion of the original components. Throughout the paper we use the term cycle to mean a simple closed curve within a graph, and the notation

$$
J \nabla L=\overline{(J \cup L)-(J \cap L)}
$$

for the closure of the symmetric difference.

Lemma 1. Let $K_{p}$ be embedded in $\mathbb{R}^{3}$ in such a way that it contains a link with components $J_{1}, \ldots, J_{n^{2}}$ and $X_{1}, \ldots, X_{n^{2}}$, and $\omega\left(J_{i}, X_{i}\right)=1$ for every $i \leq n^{2}$. Then there is a cycle $Z$ in $K_{p}$ with vertices on $J_{1} \cup \cdots \cup J_{n^{2}}$, and an index set $I$ with $|I| \geq n / 2$, such that $\omega\left(Z, X_{j}\right)=1$ for all $j \in I$.

Proof. We begin by creating a cycle $C$ in $K_{p}$ which cyclicly joins the $J_{i}$ as follows. For each $i=1, \ldots, n^{2}$, let $u_{i}$ and $w_{i}$ be vertices on the cycle $J_{i}$, 


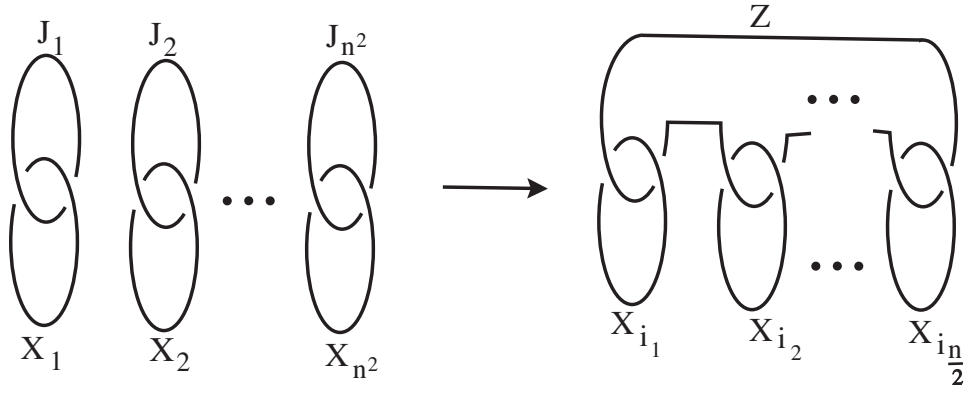

Fig. 1. Illustration of Lemma 1

and let $q_{i}$ be a path on $J_{i}$ from $u_{i}$ to $w_{i}$. For $i=1, \ldots, n^{2}-1$, let $e_{i}$ be the edge in $K_{p}$ from $w_{i}$ to $u_{i+1}$, and let $e_{n^{2}}$ be the edge in $K_{p}$ from $w_{n^{2}}$ to $u_{1}$. Let $C$ be the cycle $\bigcup_{i=1}^{n^{2}} q_{i} \cup e_{i}$. Observe that if at least $n / 2$ of the $\omega\left(C, X_{j}\right)$ 's are equal to 1 , then we are done by letting $Z=C$. So we shall assume that fewer than $n / 2$ of the $\omega\left(C, X_{j}\right)$ 's are equal to 1 .

We create an $n^{2} \times n^{2}$ matrix $M$ with entries in $\mathbb{Z}_{2}$ as follows. For each $i, j=1, \ldots, n^{2}$, let the entry $M_{i j}$ equal $\omega\left(J_{i}, X_{j}\right)$. By hypothesis, for each $i$, $M_{i i}=1$. Using row reduction mod 2, let $M^{\prime}$ denote the reduced row-echelon form of $M$. Observe that since every column of $M$ contains a 1, every column of $M^{\prime}$ also contains a 1 .

Next we will add together rows of $M^{\prime}$ as follows to create a row vector $V$, at least $n$ of whose entries are 1's. Let $r$ denote the rank of $M^{\prime}$ over $\mathbb{Z}_{2}$. First suppose that $r \geq n$. In this case, let $V$ be the vector obtained by adding together all of the non-trivial rows of $M^{\prime}$ modulo 2. Then $V$ has at least $r \geq n$ entries which are 1's. So $V$ is the desired vector. On the other hand, suppose that $r<n$. Then $n^{2} / r>n$. Observe that $M^{\prime}$ contains $r$ non-trivial rows and has $n^{2}$ columns which contain 1's. So by the Pigeonhole Principle, some row of $M^{\prime}$ has at least $n^{2} / r>n$ entries which are 1's. In this case, let $V$ be the vector representing this row.

In either case, $V$ can be written as the sum of some of the rows of $M$, say rows $i_{1}, \ldots, i_{k}$. Thus, for each $j=1, \ldots, n^{2}$, the $j$ th entry of the vector $V$ is $V_{j}=\omega\left(J_{i_{1}}, X_{j}\right)+\cdots+\omega\left(J_{i_{k}}, X_{j}\right) \bmod 2$. Recall that $V$ was chosen so that at least $n$ of the $V_{j}$ 's are equal to 1 . Also we assumed that fewer than $n / 2$ of the $\omega\left(C, X_{j}\right)$ 's are equal to 1 . Let $I$ denote the subset of $\left\{1, \ldots, n^{2}\right\}$ such that for each $j \in I$, we have simultaneously $V_{j}=1$ and $\omega\left(C, X_{j}\right)=0$. Then $|I|>n-n / 2=n / 2$. Now let $Z=C \nabla J_{i_{1}} \nabla \cdots \nabla J_{i_{k}}$. Then for each $j \in I, \omega\left(Z, X_{j}\right)=1$, as required.

Recall the following definition from graph theory. The complete bipartite graph, $K_{m, n}$, is defined as the graph whose vertices are partitioned into subsets $P_{1}$ and $P_{2}$, where $P_{1}$ contains $m$ vertices, $P_{2}$ contains $n$ vertices, and 
there is an edge between two vertices if and only if one vertex is in $P_{1}$ and the other is in $P_{2}$.

Proposition 1. For every $n$, there is a graph $G$ such that every embedding of $G$ in $\mathbb{R}^{3}$ contains a link whose mod 2 linking pattern contains the complete bipartite graph $K_{n, n}$.

Proof. Let $n$ be given. It was shown in Fleming and Diesl [5, Lemma 2.3] that there exists some $p$ such that every embedding of $K_{p}$ in $\mathbb{R}^{3}$ contains a mod 2 generalized keyring link with a ring and $n$ keys. Observe that the existence of such a $p$ also follows from our Lemma 1 together with Conway and Gordon's [1] result that every embedding of $K_{6}$ contains a link $J \cup X$ such that $\omega(J, X)=1$.

Let $m=(4 n)^{2^{n}} / 4$. Every embedding of $K_{m p}$ in $\mathbb{R}^{3}$ contains $m$ disjoint mod 2 generalized keyring links each with a ring and $n$ keys. We will prove that every embedding of $K_{m p}$ in $\mathbb{R}^{3}$ contains a link whose mod 2 linking pattern contains $K_{n, n}$.

Let $K_{m p}$ be embedded in $\mathbb{R}^{3}$. Let $X_{1}, \ldots, X_{m}$ denote the rings of the generalized keyring links in the $m$ disjoint copies of $K_{p}$ in $K_{m p}$. For each $i \leq m$ and $j \leq n$, let $J_{i j}$ be a key on the ring $X_{i}$.

Since $\omega\left(J_{i 1}, X_{i}\right)=1$ for all $i=1, \ldots, m$, we can apply Lemma 1 to the link in $K_{m p}$ with components $J_{11}, J_{21}, \ldots, J_{m 1}$ and $X_{1}, \ldots, X_{m}$. This gives us a cycle $Z_{1}$ with vertices on $J_{11} \cup \cdots \cup J_{m 1}$ and an index set $I_{1}$ with $\left|I_{1}\right| \geq \sqrt{m} / 2=(4 n)^{2^{n-1}} / 4=r_{1}$ such that for each $i \in I_{1}, \omega\left(Z_{1}, X_{i}\right)=1$. Now since $\omega\left(J_{i 2}, X_{i}\right)=1$ for all $i \in I_{1}$, we can apply Lemma 1 to the link in $K_{m p}$ whose components are all those $J_{i 2}$ and $X_{i}$ with $i \in I_{1}$. This gives us a cycle $Z_{2}$ with vertices on $J_{12} \cup \cdots \cup J_{m 2}$ and an index set $I_{2} \subseteq I_{1}$ with $\left|I_{2}\right| \geq \sqrt{r_{1}} / 2=(4 n)^{2^{n-2}} / 4=r_{2}$ such that for each $i \in I_{2}, \omega\left(Z_{2}, X_{i}\right)=1$. Continue this process to get disjoint cycles $Z_{1}, \ldots, Z_{n}$ in $K_{m p}$ and index sets $I_{n} \subseteq \cdots \subseteq I_{1}$ with $\left|I_{n}\right| \geq \sqrt{r_{n-1}} / 2=(4 n)^{2^{n-n}} / 4=n$ such that for every $i \in I_{n}$ and every $j \leq n, \omega\left(Z_{j}, X_{i}\right)=1$.

Thus the mod 2 linking pattern of the link with components $Z_{1}, \ldots, Z_{n}$ and all those $X_{i}$ with $i \in I_{n}$ contains $K_{n, n}$. Hence every embedding of $K_{m p}$ in $\mathbb{R}^{3}$ contains a link whose mod 2 linking pattern contains $K_{n, n}$.

Before we prove Theorem 1, we need one more lemma that allows us to combine components of distinct links.

Lemma 2. Let $\lambda \in \mathbb{N}$. Let $K_{p}$ be embedded in $\mathbb{R}^{3}$ in such a way that it contains a link with oriented components $J_{1}, \ldots, J_{r}, L_{1}, \ldots, L_{q}, X_{1}, \ldots, X_{m}$, and $Y_{1}, \ldots, Y_{n}$, where $r \geq m(2 \lambda+1) 2^{m}$ and $q \geq(m+n)(2 \lambda+1) 3^{m} 2^{n}$, and for any $i, j, \alpha, \beta, \operatorname{lk}\left(J_{i}, X_{\alpha}\right) \neq 0$ and $\operatorname{lk}\left(L_{j}, Y_{\beta}\right) \neq 0$. Then $K_{p}$ contains a cycle $Z$ with vertices in $J_{1} \cup \cdots \cup J_{r} \cup L_{1} \cup \cdots \cup L_{q}$ such that for all $\alpha$ and $\beta$, $\left|\operatorname{lk}\left(Z, X_{\alpha}\right)\right|>\lambda$ and $\left|\operatorname{lk}\left(Z, Y_{\beta}\right)\right|>\lambda$. 
Proof. First we consider the signs of the linking numbers $\operatorname{lk}\left(J_{i}, X_{1}\right)$ for $i \leq r$. At least half of these linking numbers have the same sign. Thus without loss of generality, $J_{1}, \ldots, J_{\lceil r / 2\rceil}$ all have the same sign linking number with $X_{1}$. Furthermore, we may assume these linking numbers are all positive (otherwise, simply reverse the orientation of $X_{1}$ ). Now, consider the signs of the linking numbers $\operatorname{lk}\left(J_{i}, X_{2}\right)$ for $i \leq\lceil r / 2\rceil$. At least half of these have the same sign. We continue this process for each subsequent $X_{\alpha}$. In this way, we end up with cycles $J_{1}, \ldots, J_{r^{\prime}}$ which each have positive linking number with every $X_{\alpha}$, and $r^{\prime} \geq r / 2^{m} \geq m(2 \lambda+1)$. From now on the only $J_{i}$ that we consider will be $J_{1}, \ldots, J_{m(2 \lambda+1)}$, which all have positive linking number with every $X_{\alpha}$.
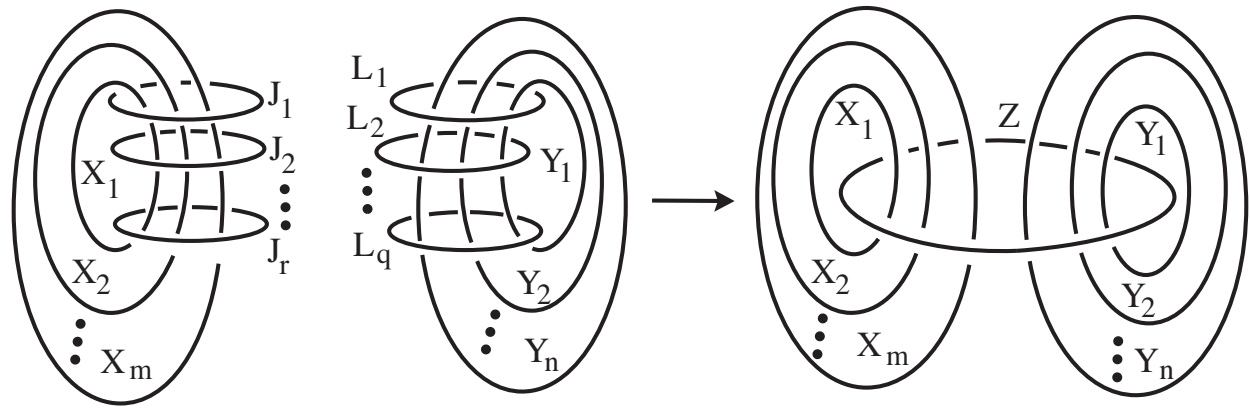

Fig. 2. Illustration of Lemma 2

Next we consider the signs of the linking numbers $\operatorname{lk}\left(L_{j}, Y_{1}\right)$ for $j \leq q$. By using an argument analogous to the one above, we end up with cycles $L_{1}, \ldots, L_{q^{\prime}}$ which each have positive linking number with every $Y_{\beta}$, and $q^{\prime} \geq q / 2^{n} \geq(m+n)(2 \lambda+1) 3^{m}$.

Now we consider the signs (positive, negative, or zero) of the linking numbers $\operatorname{lk}\left(L_{j}, X_{1}\right)$ for $j \leq q^{\prime}$. By the Pigeonhole Principle at least onethird of these signs are the same. Without loss of generality, we can assume that $L_{1}, \ldots, L_{\left\lceil q^{\prime} / 3\right\rceil}$ each have the same sign (positive, negative, or zero) linking number with $X_{1}$. We continue this process for each subsequent $X_{\alpha}$. In this way, we end up with cycles $L_{1}, \ldots, L_{q^{\prime \prime}}$ which each have the same sign (positive, negative, or zero) linking number with every $X_{\alpha}$, and $q^{\prime \prime} \geq$ $q^{\prime} / 3^{m} \geq(m+n)(2 \lambda+1)$. From now on the only $L_{j}$ that we consider are $L_{1}, \ldots, L_{(m+n)(2 \lambda+1)}$, which all have positive linking number with every $Y_{\beta}$ and all have the same sign linking number with every $X_{\alpha}$.

We shall create a cycle $C_{0}$ which cyclicly joins $J_{1}, \ldots, J_{m(2 \lambda+1)}$ and $L_{1}, \ldots, L_{(m+n)(2 \lambda+1)}$ as follows. For $i \leq m(2 \lambda+1)$, let $u_{i}$ and $w_{i}$ be vertices on $J_{i}$, and for $j \leq(m+n)(2 \lambda+1)$, let $u_{m(2 \lambda+1)+j}$ and $w_{m(2 \lambda+1)+j}$ be 
vertices on $L_{j}$. Also, for $k \leq(2 m+n)(2 \lambda+1)-1$, let $e_{k}$ be the edge in $K_{p}$ from $w_{k}$ to $u_{k+1}$, and let $e_{(2 m+n)(2 \lambda+1)}$ be the edge in $K_{p}$ from $w_{(2 m+n)(2 \lambda+1)}$ to $u_{1}$. Finally, for $i \leq m(2 \lambda+1)$, let $q_{i}$ be the path on $J_{i}$ from $u_{i}$ to $w_{i}$ which travels opposite to the orientation on $J_{i}$, and for $j \leq(m+n)(2 \lambda+1)$, let $q_{m(2 \lambda+1)+j}$ be the path on $L_{j}$ from $u_{m(2 \lambda+1)+j}$ to $w_{m(2 \lambda+1)+j}$ which travels opposite to the orientation of $L_{j}$. Now let

$$
C_{0}=\bigcup_{k=1}^{(2 m+n)(2 \lambda+1)} e_{k} \cup q_{k}
$$

with the induced orientation. Also for each $s \leq m(2 \lambda+1)$, let

$$
C_{s}=C_{0} \nabla J_{1} \nabla \cdots \nabla J_{s} .
$$

Observe that for every $\alpha$ and $s \leq m(2 \lambda+1)-1, \operatorname{lk}\left(C_{s+1}, X_{\alpha}\right)=$ $\operatorname{lk}\left(C_{s}, X_{\alpha}\right)+\operatorname{lk}\left(J_{s+1}, X_{\alpha}\right)$ and $\operatorname{lk}\left(J_{s+1}, X_{\alpha}\right)>0$. Hence for a given $\alpha$, $\mathrm{lk}\left(C_{s}, X_{\alpha}\right)$ is a strictly increasing function of $s$. In particular, for a given $\alpha$, the values of $\operatorname{lk}\left(C_{s}, X_{\alpha}\right)$ are distinct for different values of $s$. As there are $2 \lambda+1$ distinct values of $a$ with $|a| \leq \lambda$, for a given $\alpha$ there are at most $2 \lambda+1$ values of $s$ such that $\left|\operatorname{lk}\left(C_{s}, X_{\alpha}\right)\right| \leq \lambda$. Since there are $m$ values of $\alpha$, there are at most $m(2 \lambda+1)$ values of $s$ such that there is an $\alpha$ with $\left|\operatorname{lk}\left(C_{s}, X_{\alpha}\right)\right| \leq \lambda$. Now by the Pigeonhole Principle, since there are $m(2 \lambda+1)+1$ values of $s$ (including $s=0$ ), there must be at least one $C_{s}$ such that $\left|\operatorname{lk}\left(C_{s}, X_{\alpha}\right)\right|>\lambda$ for every $\alpha$. Let $D_{0}$ denote such a $C_{s}$.

Let us recall that each $Y_{\beta}$ has positive linking number with $L_{1}, \ldots$, $L_{(m+n)(2 \lambda+1)}$, and each $X_{\alpha}$ has the same sign (positive, negative or zero) linking number with $L_{1}, \ldots, L_{(m+n)(2 \lambda+1)}$. In fact, by changing the orientation of some $X_{\alpha}$, we can assume that each $X_{\alpha}$ has non-negative linking number with $L_{1}, \ldots, L_{(m+n)(2 \lambda+1)}$. Note that changing the orientation of a particular $X_{\alpha}$ does not change the fact that $\left|\operatorname{lk}\left(D_{0}, X_{\alpha}\right)\right|>\lambda$. Now let $S$ denote the set of all the $Y_{\beta}$ 's together with those $X_{\alpha}$ 's which have positive linking number with all of $L_{1}, \ldots, L_{(m+n)(2 \lambda+1)}$. For each $t \leq(m+n)(2 \lambda+1)$, let

$$
D_{t}=D_{0} \nabla L_{1} \nabla \cdots \nabla L_{t} .
$$

Since $\alpha \leq m$ and $\beta \leq n$, the set $S$ contains at most $m+n$ cycles. Also, each cycle in $S$ has positive linking number with $L_{1}, \ldots, L_{(m+n)(2 \lambda+1)}$. Thus we can use the same argument as the one we used for the $C_{s}$ to show that there is some $D_{t}$ such that $\left|\operatorname{lk}\left(D_{t}, A\right)\right|>\lambda$ for each $A \in S$. Let $Z$ denote such a $D_{t}$. Finally, observe that for each $X_{\alpha}$ not in $S, \operatorname{lk}\left(L_{j}, X_{\alpha}\right)=0$ for all $j \leq(m+n)(2 \lambda+1)$. Hence if $X_{\alpha} \notin S$, then $\left|\operatorname{lk}\left(Z, X_{\alpha}\right)\right|=\left|\operatorname{lk}\left(D_{0}, X_{\alpha}\right)\right|>\lambda$. So, for every $\alpha$ and $\beta,\left|\operatorname{kk}\left(Z, X_{\alpha}\right)\right|>\lambda$ and $\left|\operatorname{lk}\left(Z, Y_{\beta}\right)\right|>\lambda$ as desired.

In the proof of Theorem 1, we will use Lemma 2 together with the following definition from graph theory. A complete m-partite graph is defined 
as a graph whose vertices are partitioned into subsets $P_{1}, \ldots, P_{m}$ and there is an edge between two vertices if and only if the two vertices are in distinct subsets of the partition.

Theorem 1. Let $\lambda \in \mathbb{N}$. For every $n \in \mathbb{N}$, there is a graph $G$ such that every embedding of $G$ in $\mathbb{R}^{3}$ contains a link whose linking pattern is $K_{n}$ with every weight at least $\lambda$.

Proof. For all $m, n \in \mathbb{N}$, let $H(n, m)$ denote the complete $(n+2)$-partite graph with partitions $P_{1}$ and $P_{2}$ containing $m$ vertices each and partitions $Q_{1}, \ldots, Q_{n}$ each containing a single vertex. We will prove by induction on $n$ that for each $n \geq 0$, for every $m \geq 1$, there is a graph $G$ such that every embedding of $G$ in $\mathbb{R}^{3}$ contains a link whose weighted linking pattern contains $H(n, m)$, and the weight of every edge between vertices in $Q_{i}$ and $Q_{j}$ is greater than $\lambda$.

The base case is for $n=0$. Observe that $H(0, m)$ is the complete bipartite graph $K_{m, m}$. Hence by Proposition 1 we know that, for every $m$, there is a graph $G$ such that every embedding of $G$ in $\mathbb{R}^{3}$ contains a link whose linking pattern contains $H(0, m)$.

As our induction hypothesis we suppose that for some $n \geq 0$, for every $m \geq 1$ there is a graph $G$ such that every embedding of $G$ in $\mathbb{R}^{3}$ contains a link whose linking pattern contains $H(n, m)$, and the weight of every edge between vertices in $Q_{i}$ and $Q_{j}$ is greater than $\lambda$. Let $m$ be given. Let $q=$ $(2 m+n)(2 \lambda+1) 3^{m} 2^{m+n}$ and let $s=m+q$. Consider the graph $H(n, s)$ with partitions $P_{1}, P_{2}, Q_{1}, \ldots, Q_{n}$, where the vertices in each partition are denoted as follows. The $s$ vertices in $P_{1}$ are denoted by $X_{1}, \ldots, X_{m}, L_{1}, \ldots, L_{q}$. The $s$ vertices in $P_{2}$ are denoted by $Y_{1}, \ldots, Y_{m}, J_{1}, \ldots, J_{q}$. For each $i \leq n$, the partition $Q_{i}$ contains a single vertex denoted by $Y_{m+i}$.

It follows from our inductive hypothesis that there is a graph $G$ such that every embedding of $G$ in $\mathbb{R}^{3}$ contains a link $L$ whose linking pattern contains $H(n, s)$, and for any $i \neq j$ the weight of the edge between vertices $Y_{m+i}$ and $Y_{m+j}$ is greater than $\lambda$. Without loss of generality, $G$ is a complete graph $K_{p}$. Let $K_{p}$ be embedded in $\mathbb{R}^{3}$. We will prove that $K_{p}$ also contains a link whose weighted linking pattern contains $H(n+1, m)$ with the desired weights. We shall abuse notation and let each of the components of the link $L$ in $K_{p}$ be denoted by the name of the vertex that represents that component in the linking pattern described above.

We can apply Lemma 2 to the oriented link with components $J_{1}, \ldots, J_{q}$, $L_{1}, \ldots, L_{q}, X_{1}, \ldots, X_{m}$, and $Y_{1}, \ldots, Y_{m+n}$ in $K_{p}$ with

$$
r=q=(2 m+n)(2 \lambda+1) 3^{m} 2^{m+n},
$$

to get a cycle $Y_{m+n+1}$ with vertices in $J_{1} \cup \cdots \cup J_{q} \cup L_{1} \cup \cdots \cup L_{q}$ such that for every $\alpha \leq n$ and $\beta \leq m+n,\left|\operatorname{lk}\left(Y_{m+n+1}, X_{\alpha}\right)\right|>\lambda$ and $\left|\operatorname{lk}\left(Y_{m+n+1}, Y_{\beta}\right)\right|>\lambda$. 
Thus $K_{p}$ contains a link $L^{\prime}$ with components $X_{1}, \ldots, X_{m}, Y_{1}, \ldots, Y_{m+n+1}$. The components of $L^{\prime}$ can be partitioned into subsets $P_{1}^{\prime}, P_{2}^{\prime}, Q_{1}^{\prime}, \ldots, Q_{n+1}^{\prime}$, where $P_{1}^{\prime}$ contains $X_{1}, \ldots, X_{m}, P_{2}^{\prime}$ contains $Y_{1}, \ldots, Y_{m}$, and for each $i=$ $1, \ldots, n+1, Q_{i}^{\prime}$ contains $Y_{m+i}$. Furthermore, every component in one partition is linked with every component in all the other partitions. Also, for all $i, j \leq n+1$ with $i \neq j,\left|\operatorname{lk}\left(Y_{m+i}, Y_{m+j}\right)\right|>\lambda$. It follows that the weighted linking pattern of $L^{\prime}$ contains $H(n+1, m)$, and the weight of every edge between vertices $Q_{i}^{\prime}$ and $Q_{j}^{\prime}$ is greater than $\lambda$.

Thus, we have shown that for every $n \geq 0$ and $m \geq 1$, there is a graph $G$ such that every embedding of $G$ in $\mathbb{R}^{3}$ contains a link whose weighted linking pattern contains $H(n, m)$, and the weight of every edge between vertices $Y_{m+i}$ and $Y_{m+j}$ in $Q_{i}$ and $Q_{j}$ respectively is greater than $\lambda$. Observe that the subgraph of $H(n, m)$ consisting of the vertices $Y_{m+1}, \ldots, Y_{m+n}$ in $Q_{1}, \ldots, Q_{n}$ respectively, together with the edges between them, is the complete graph $K_{n}$. Hence every embedding of $G$ in $\mathbb{R}^{3}$ contains a link whose linking pattern is $K_{n}$, and the weight of every edge of $K_{n}$ is greater than $\lambda$.

The following proposition shows that every graph $G$ has some embedding in $\mathbb{R}^{3}$ such that the linking pattern of every link in that embedding is a complete graph. Hence complete graphs are the only linking patterns which have the property described by Theorem 1 .

Proposition 2. Given any $\lambda>0$ and any graph $G$, there exists an embedding $G^{\prime}$ of $G$ in $\mathbb{R}^{3}$ and an orientation of the cycles in $G^{\prime}$ such that for every pair of disjoint oriented cycles $C_{1}$ and $C_{2}$ in $G^{\prime}, \operatorname{lk}\left(C_{1}, C_{2}\right) \geq \lambda$.

Proof. We start with any embedding of the graph $G$ in $\mathbb{R}^{3}$. We arbitrarily assign to each edge a unique positive integer which will be the weight of that edge. The weights give the set of edges of $G$ a linear ordering. We put an arbitrary orientation on each edge, and then orient each cycle according to the orientation of its edge whose weight is the largest. For each pair $\left(e_{i}, f_{i}\right)$ of disjoint edges we denote the smaller weighted edge by $e_{i}$ and the larger weighted edge by $f_{i}$. Note that a given edge may occur in multiple pairs, sometimes as the smaller weighted edge and sometimes as the larger weighted edge. Let $\left(e_{0}, f_{0}\right), \ldots,\left(e_{n}, f_{n}\right)$ denote the set of all pairs of disjoint edges ordered lexicographically from smallest weighted pair to largest weighted pair. We describe as follows how to add twists between each pair of edges so as to obtain the desired embedding of the graph in $\mathbb{R}^{3}$.

Let $M=\max \left\{\left|\operatorname{lk}\left(C_{1}, C_{2}\right)\right|\right\}$ with the maximum taken over all pairs of disjoint cycles $C_{1}$ and $C_{2}$ in $G$, and let $t=M+\lambda$.

For each $i$, we choose an arc $a_{i}$ with one endpoint in the interior of $e_{i}$ and the other endpoint in the interior of $f_{i}$ so that $a_{i}$ is otherwise disjoint from $G$. In addition, we require that the $\operatorname{arcs} a_{0}, \ldots, a_{n}$ be pairwise disjoint 
with neighborhoods that are also pairwise disjoint. For each $i=0, \ldots, n$, we change the embedding of $e_{i}$ and $f_{i}$ by adding $3^{i} t$ positive full twists between them within the neighborhood of $a_{i}$ (see Figure 3). Note that a given edge will occur in multiple pairs $\left(e_{i}, f_{i}\right)$ and hence will be changed within the disjoint neighborhoods of multiple $a_{i}$ 's.
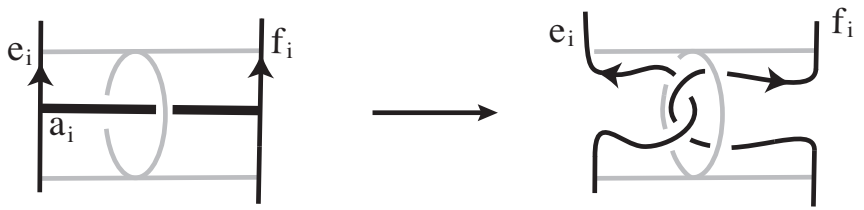

Fig. 3. We add $3^{i} t$ positive full twists between $e_{i}$ and $f_{i}$ within a tubular neighborhood of $a_{i}$

Adding these twists gives us a new embedding $G^{\prime}$ of $G$. Fix a pair of disjoint cycles $C_{1}$ and $C_{2}$ in $G$, and let $C_{1}^{\prime}$ and $C_{2}^{\prime}$ denote the corresponding pair of cycles in $G^{\prime}$. Observe that any crossings between pairs of $a_{j}$ 's or between an $a_{j}$ and an $e_{i}$ or $f_{i}$ that added new crossings between $C_{1}^{\prime}$ and $C_{2}^{\prime}$ occur in pairs with opposite sign since we added an integer number of full twists between $e_{i}$ and $f_{i}$. Hence such crossings do not contribute to the value of $\operatorname{lk}\left(C_{1}^{\prime}, C_{2}^{\prime}\right)$. Thus $\operatorname{lk}\left(C_{1}^{\prime}, C_{2}^{\prime}\right)=\operatorname{lk}\left(C_{1}, C_{2}\right)+\varepsilon_{0} t+\cdots+\varepsilon_{n} 3^{n} t$, where $\varepsilon_{i}$ equals 0 or \pm 1 depending on which edges $e_{i}$ and $f_{i}$ are in $C_{1}$ and $C_{2}$, and on whether the twists we have added between $e_{i}$ and $f_{i}$ induce negative or positive twists between $C_{1}$ and $C_{2}$.

Pick the largest $k$ such that $\varepsilon_{k} \neq 0$. Clearly $k \geq 1$. Then the pair $\left(e_{k}, f_{k}\right)$ is lexicographically the largest weighted pair with one edge in $C_{1}$ and the other in $C_{2}$. Therefore the orientations of $C_{1}$ and $C_{2}$ are induced by those of $e_{k}$ and $f_{k}$. Since we have added $3^{k} t$ positive full twists between $e_{k}$ and $f_{k}$, it follows that $\varepsilon_{k}=1$. Thus we have

$$
\begin{aligned}
\operatorname{lk}\left(C_{1}^{\prime}, C_{2}^{\prime}\right) & \geq-M-\left(1+3+\cdots+3^{k-1}\right) t+3^{k} t \\
& =3^{k} t-\left(3^{k}-1\right) t /(3-1)-M=\left(3^{k}+1\right) t / 2-M \\
& =\left(3^{k}+1\right)(M+\lambda) / 2-M \geq \lambda .
\end{aligned}
$$

Since the choice of the pair of cycles $C_{1}$ and $C_{2}$ was arbitrary this proves the proposition.

We saw in Theorem 1 that for any $\lambda>0$ there is a graph $G$ such that every embedding of $G$ contains a link whose linking pattern is $K_{n}$ with every weight at least $\lambda$. Recall that the weight of an edge in the linking pattern is defined as the absolute value of the linking number of the associated link in the embedded graph. It is natural to wonder whether we can remove the absolute value from the conclusion of Theorem 1 . That is, we would like to 
know if is there a graph $G$ such that every embedding of $G$ in $\mathbb{R}^{3}$ contains a link with components $L_{1}, \ldots, L_{n}$ which can be oriented in such a way that $\operatorname{lk}\left(L_{i}, L_{j}\right) \geq \lambda$ for all $i \neq j$. We now prove Corollary 1 which shows that the answer to this question is no.

Corollary 1. Let $G$ be a graph. There is some embedding $G^{\prime}$ of $G$ in $\mathbb{R}^{3}$ such that for any three disjoint cycles in $G^{\prime}$, no matter how they are oriented, at least one of the three pairs of oriented cycles will have positive linking number; and there is some embedding $G^{\prime \prime}$ of $G$ in $\mathbb{R}^{3}$ such that for any three disjoint cycles in $G^{\prime \prime}$, no matter how they are oriented, at least one of the three pairs of oriented cycles will have negative linking number.

Proof. We begin with the embedding $G^{\prime}$ and the orientation of all of the cycles of $G^{\prime}$ which is given by Proposition 2. Thus for any pair of oriented cycles $C_{1}^{\prime}$ and $C_{2}^{\prime}$ in $G^{\prime}, \operatorname{lk}\left(C_{1}^{\prime}, C_{2}^{\prime}\right) \geq \lambda>0$. Now, consider any three disjoint cycles in $G^{\prime}$. With the given orientations, their three pairwise linking numbers are all positive. If we change the orientation of one of the three cycles, then the linking number between the two unchanged cycles remains positive. If we change the orientation of two of the three cycles, then the linking number between the two changed cycles remains positive. Finally, if we change the orientation of all three cycles, then all three linking numbers remain positive. Thus no matter how they are oriented, at least one pair will have positive linking number.

Now let $G^{\prime \prime}$ denote the mirror image of $G^{\prime}$. It follows that for any three disjoint cycles in $G^{\prime \prime}$ and every orientation of these three cycles at least one of the three will have negative linking number.

Thus we have shown that there is no graph whose every embedding contains a link of three cycles where for some orientation of the cycles all the linking numbers are positive, and there is no graph whose every embedding contains a link of three cycles where for some orientation of the cycles all the linking numbers are negative. By contrast, the next proposition shows that there is a graph whose every embedding contains a link of three cycles (indeed, of $n$ cycles for any given $n \geq 3$ ) such that for any orientation of the cycles all the linking numbers have the same sign (either positive or negative). However, the sign (as well as the cycles) will depend on the specific embedding of the graph in $\mathbb{R}^{3}$.

Proposition 3. Given any $n \in \mathbb{N}$, there exists an $r$ such that every embedding of $K_{r}$ in $\mathbb{R}^{3}$, with an arbitrary orientation assigned to every cycle, contains an n-component link such that the linking numbers of every pair of components in the oriented link are either all positive or all negative. 
Proof. By Ramsey theory [6], there is an $m$ such that for any 2-coloring of the edges of $K_{m}$ with red or blue, there is a subgraph $K_{n}$ whose edges are all red or all blue. We know by Theorem 1 that, given the above $m$, every sufficiently large $K_{r}$ embedded in $\mathbb{R}^{3}$ contains an $m$-component link whose linking pattern is $K_{m}$. Given an embedding of this $K_{r}$ in $\mathbb{R}^{3}$, we put an arbitrary orientation on each of the $m$ components of the link in this embedding. Then we color each edge in the associated linking pattern $K_{m}$ red or blue according to whether that edge corresponds to a positive or negative linking number respectively. Now we know there is a subgraph $K_{n}$ of this colored $K_{m}$ whose edges are all red or all blue. This means that for the $n$-component oriented link corresponding to this monocrhromatic $K_{n}$ linking pattern, the pairwise linking numbers are either all positive or all negative.

3. Intrinsic knotting of the components. We extend our definition of weighted linking pattern to include knotted components as follows.

Definition 3. Given an oriented link $L$ with components $L_{1}, \ldots, L_{n}$, the weighted knotting and linking pattern of $L$ is the weighted linking pattern of $L$ together with a weight assigned to each vertex $v_{i}$ representing the value of $\left|a_{2}\left(L_{i}\right)\right|$.

In light of Theorem 1, it is natural to ask whether for any weighted knotting and linking pattern $\Gamma$, there is a graph $G$ such that every embedding of $G$ in $\mathbb{R}^{3}$ contains a link whose weighted knotting and linking pattern is at least as "complex" as $\Gamma$. The goal of this section is to prove that this is indeed the case. In particular, we will prove the following restatement of Theorem 2.

Theorem 2. Let $\alpha \in \mathbb{N}$. For every $n \in \mathbb{N}$, there is a graph $G$ such that every embedding of $G$ in $\mathbb{R}^{3}$ contains a link whose linking pattern is $K_{n}$ with every edge weight and vertex weight at least $\alpha$.

We begin with some preliminary results. In the following lemma we shall use the notation $A \nabla \varepsilon B$ where $\varepsilon \in\{0,1\}$. If $\varepsilon=1$, we shall mean $A \nabla B$. If $\varepsilon=0$, we shall mean $A \nabla \emptyset=A$.

Lemma 3. Let $\lambda>0$, and let $A_{1}, \ldots, A_{n}$ and $B_{1}, \ldots, B_{6 n+6}$ be disjoint oriented cycles in a complete graph $K_{r}$ embedded in $\mathbb{R}^{3}$ such that $\operatorname{lk}\left(A_{h}, B_{i}\right) \geq \lambda$ for all $h$ and $i$. Then there exist disjoint cycles $C_{1}, C_{2}, C_{3}, C_{4}$ $\in\left\{B_{i}\right\}$ and an oriented cycle $W^{\prime}$ in $K_{r}$ with vertices on $\bigcup_{i} B_{i}$ such that $W^{\prime}$ intersects each of $C_{1}, C_{2}, C_{3}, C_{4}$ in exactly one arc with orientation opposite that of each $C_{i}$, and for every $h$,

$$
\left|\operatorname{lk}\left(A_{h}, W^{\prime} \nabla \varepsilon_{1} C_{1} \nabla \varepsilon_{2} C_{2} \nabla \varepsilon_{3} C_{3} \nabla \varepsilon_{4} C_{4}\right)\right| \geq \lambda
$$

for every choice of $\varepsilon_{1}, \ldots, \varepsilon_{4} \in\{0,1\}$. 
Proof. On each oriented cycle $B_{i}$, pick adjacent vertices $x_{i}$ and $y_{i}$ so that the orientation induced on the edge $\beta_{i}=\left\{x_{i}, y_{i}\right\}$ goes from $x_{i}$ to $y_{i}$. Let $W_{0}$ be the cycle which is the union of the edges $\beta_{i}$ and the edges $\left\{y_{i}, x_{i+1}\right\}, 1 \leq i \leq 6 n+5$, and $\left\{y_{6 n+6}, x_{1}\right\}$. Orient $W_{0}$ so that on each $\beta_{i}$ the orientation goes from $y_{i}$ to $x_{i}$. For the remainder of this proof, we relabel $B_{1}, \ldots, B_{6 n+6}$ as $B_{1}^{1}, \ldots, B_{1}^{6}, B_{2}^{1}, \ldots, B_{2}^{6}, \ldots, B_{n+1}^{1}, \ldots, B_{n+1}^{6}$. Figure 4 illustrates the cycles $B_{1}^{1}, \ldots, B_{1}^{6}, B_{2}^{1}, \ldots, B_{2}^{6}, \ldots, B_{n+1}^{1}, \cdots, B_{n+1}^{6}$ together with $W_{0}$.

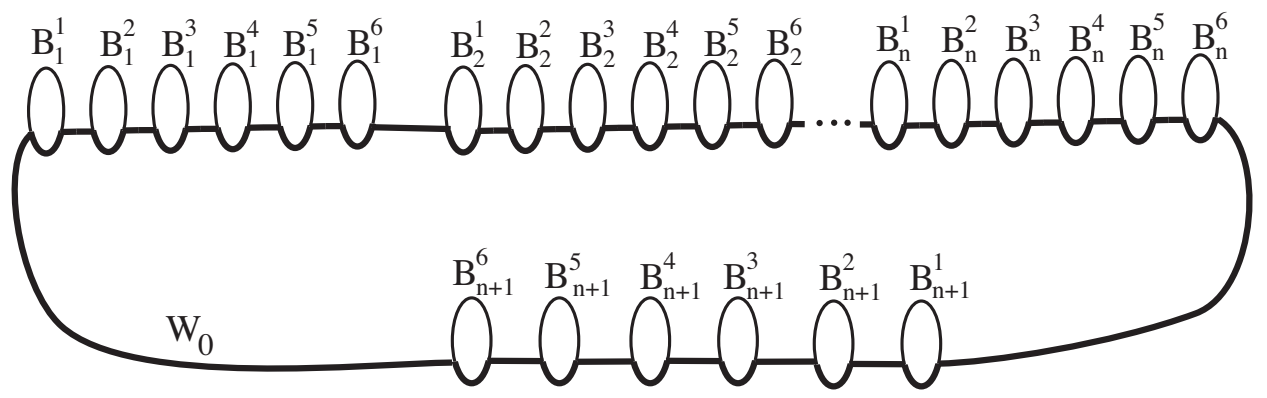

Fig. 4. $B_{1}^{1}, \ldots, B_{1}^{6}, B_{2}^{1}, \ldots, B_{2}^{6}, \ldots, B_{n+1}^{1}, \ldots, B_{n+1}^{6}$ together with $W_{0}$

For $1 \leq i \leq n$, let $W_{i}=W_{i-1} \nabla B_{i}^{1} \nabla \cdots \nabla B_{i}^{6}$. We have two cases:

CASE 1: For some $r \geq 0, \operatorname{lk}\left(W_{r}, A_{h}\right) \geq 0$ for all $h$. Then letting $W^{\prime}=$ $W_{r} \nabla B_{r+1}^{6}$ and $C_{j}=B_{r+1}^{j}$ for $j=1,2,3,4$ gives us the desired result.

CASE 2: For every $k \geq 0, \operatorname{lk}\left(W_{k}, A_{h}\right)<0$ for some $h$. In this case, for each $k$ let $n(k)$ be the number of $A_{h}$ 's for which $\operatorname{lk}\left(W_{k}, A_{h}\right)<0$. Then $1 \leq n(k) \leq n$ for each $k=0,1, \ldots, n$. Since there are $n+1$ values of $k$, by the Pigeonhole Principle, $n(r)=n\left(r^{\prime}\right)$ for some $r$ and $r^{\prime}$. Without loss of generality, $r<r^{\prime}$. We see as follows that $n(k)$ is a non-increasing function of $k$. For each $h$ and $k, \operatorname{lk}\left(W_{k}, A_{h}\right)>\operatorname{lk}\left(W_{k-1}, A_{h}\right)$ since $\operatorname{lk}\left(B_{k}^{j}, A_{h}\right) \geq \lambda>0$ for all $j$. It follows that $n(r)=n(r+1)$. So, for each $h, \operatorname{lk}\left(A_{h}, W_{r}\right)$ has the same sign as $\operatorname{lk}\left(A_{h}, W_{r+1}\right)$.

Now, as in Case 1, we let $W^{\prime}=W_{r} \nabla B_{r+1}^{6}$ and $C_{j}=B_{r+1}^{j}$ for $j=1,2,3,4$. We verify as follows that this gives us the desired result. Fix an $A_{h}$. If $\operatorname{lk}\left(A_{h}, W_{r}\right) \geq 0$, then clearly

$$
\operatorname{lk}\left(A_{h}, W^{\prime} \nabla \varepsilon_{1} C_{1} \nabla \varepsilon_{2} C_{2} \nabla \varepsilon_{3} C_{3} \nabla \varepsilon_{4} C_{4}\right) \geq \lambda
$$

for every choice of $\varepsilon_{1}, \ldots, \varepsilon_{4} \in\{0,1\}$, as desired. So suppose $\operatorname{lk}\left(A_{h}, W_{r}\right)<0$. Then $\operatorname{lk}\left(A_{h}, W_{r+1}\right)<0$, since $\operatorname{lk}\left(A_{h}, W_{r}\right)$ has the same sign as $\operatorname{lk}\left(A_{h}, W_{r+1}\right)$. 
Now, for every choice of $\varepsilon_{1}, \ldots, \varepsilon_{4} \in\{0,1\}$,

$$
\begin{aligned}
\operatorname{lk}\left(A_{h}, W^{\prime} \nabla \varepsilon_{1} C_{1} \nabla \varepsilon_{2} C_{2} \nabla \varepsilon_{3} C_{3} \nabla \varepsilon_{4} C_{4}\right) & \leq \operatorname{lk}\left(A_{h}, W^{\prime} \nabla C_{1} \nabla C_{2} \nabla C_{3} \nabla C_{4}\right) \\
& \leq \operatorname{lk}\left(A_{h}, W_{r+1}\right)-\operatorname{lk}\left(A_{h}, B_{r+1}^{5}\right)<-\lambda
\end{aligned}
$$

as desired.

Lemma 4. Let $\lambda>0$, and let $A_{1}, \ldots, A_{n}$ and $B_{1}, \ldots, B_{6 n+6}$ be disjoint oriented cycles in an embedded complete graph $K_{r}$ such that $\operatorname{lk}\left(A_{h}, B_{i}\right) \geq \lambda$ and $\left|\operatorname{lk}\left(B_{i}, B_{j}\right)\right| \geq \lambda$ for all $h, i$, and $j$. Then there exists an oriented cycle $K$ in $K_{r}$ with vertices on $\bigcup_{i} B_{i}$ such that $\left|a_{2}(K)\right| \geq \lambda^{2} / 16$ and, for every $h$, $\left|\operatorname{lk}\left(A_{h}, K\right)\right| \geq \lambda$.

Proof. Let $W^{\prime}$ and $C_{1}, C_{2}, C_{3}, C_{4}$ denote the oriented cycles given by Lemma 3. We collapse the four arcs of $W^{\prime}$ which are not in any of the $C_{i}$ to obtain the pseudograph illustrated on the right in Figure 5. We denote this pseudograph by $D_{4}$.

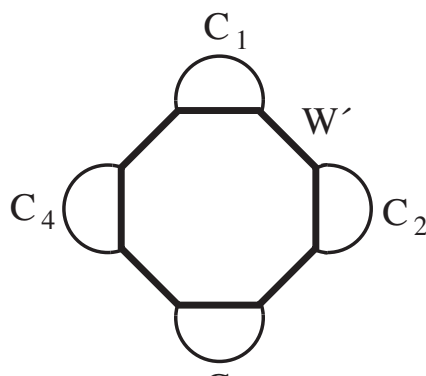

$\mathrm{C}_{3}$

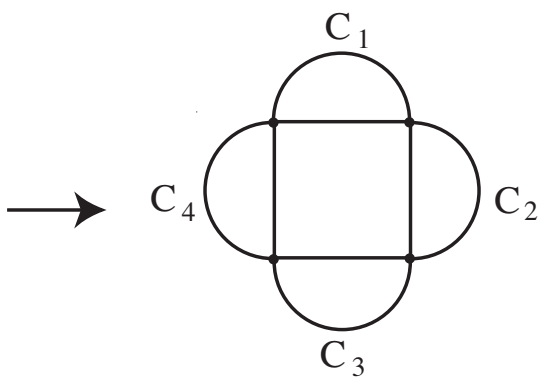

$\mathrm{C}_{3}$

Fig. 5. We collapse the four arcs of $W^{\prime}$ not in any $C_{i}$ to obtain the pseudograph $D_{4}$

Let $S$ denote the set of all Hamiltonian cycles in $D_{4}$. It follows from [9] that

$$
\sum_{Q \in S}\left|a_{2}(Q)\right| \geq\left|\operatorname{lk}\left(C_{1}, C_{3}\right) \operatorname{lk}\left(C_{2}, C_{4}\right)\right| .
$$

Since $C_{1}, C_{2}, C_{3}, C_{4} \in\left\{B_{i}\right\}$, we deduce that both $\left|\operatorname{lk}\left(C_{1}, C_{3}\right)\right| \geq \lambda$ and $\left|\operatorname{lk}\left(C_{2}, C_{4}\right)\right| \geq \lambda$. Thus $\sum_{Q \in S}\left|a_{2}(Q)\right| \geq \lambda^{2}$. As we are taking the sum of 16 non-negative integers, there must be some $Q_{0} \in S$ such that $\left|a_{2}\left(Q_{0}\right)\right| \geq$ $\lambda^{2} / 16$. Let $K$ denote the cycle of the form

$$
W^{\prime} \nabla \varepsilon_{1} C_{1} \nabla \varepsilon_{2} C_{2} \nabla \varepsilon_{3} C_{3} \nabla \varepsilon_{4} C_{4}
$$

which collapses to $Q_{0}$ when we collapse the four edges of $W^{\prime}$ not in any $C_{i}$. Then $\left|a_{2}(K)\right| \geq \lambda^{2} / 16$. Also, it follows from Lemma 3 that for every $h$, $\left|\operatorname{lk}\left(A_{h}, K\right)\right| \geq \lambda$. Thus $K$ has both of the required properties. 
Roughly speaking, our goal is to show that every sufficiently large complete spatial graph contains a link with a given large number of components, all with large pairwise linking numbers and large $a_{2}$ coefficients. The idea is that, by Theorem 1, our large complete spatial graph contains a link with a large number of components whose pairwise linking numbers have large absolute value. We would like to apply Lemma 4 repeatedly to this link, each time increasing the number of components that have large $a_{2}$ coefficients, at the expense of decreasing the total number of components in the link at hand. However, to use Lemma 4, we need positive linking numbers between the $A_{h}$ 's and the $B_{i}$ 's. We accomplish this by giving ourselves the luxury of starting out with a lot of $B_{i}$ 's and discarding those with negative linking numbers, as follows.

Lemma 5. Let $n, \lambda \in \mathbb{N}$. Suppose that a complete graph $K_{r}$ embedded in $\mathbb{R}^{3}$ contains an oriented link $L_{0}$ with $f^{n}(n)$ components, where $f(n)=n-1+$ $(6 n) 2^{n-2}$, such that the linking number of every pair of components of $L_{0}$ has absolute value at least $\lambda$. Then $K_{r}$ contains an oriented link with components $Q_{1}, \ldots, Q_{n}$ such that, for any $i \neq j,\left|\operatorname{lk}\left(Q_{i}, Q_{j}\right)\right| \geq \lambda$ and $\left|a_{2}\left(Q_{i}\right)\right| \geq \lambda^{2} / 16$.

Proof. Before we begin a recursive argument, we start by introducing some variables. For every $i=1, \ldots, n$, we let $m_{i}=f^{i-1}(n)-1$. So for each $i$,

$$
f^{i}(n)=f\left(m_{i}+1\right)=m_{i}+\left(6 m_{i}+6\right) 2^{m_{i}-1} .
$$

Now for each $i$, we let

$$
m_{i}^{\prime}=\left(6 m_{i}+6\right) 2^{m_{i}-1},
$$

so that $f^{i}(n)=m_{i}+m_{i}^{\prime}$.

We start our recursive argument with the given link $L_{0}$, which has $f^{n}(n)=m_{n}+m_{n}^{\prime}$ components. We begin by partitioning the components of $L_{0}$ into two subsets: $A_{1}, \ldots, A_{m_{n}}$ and $B_{1}, \ldots, B_{m_{n}^{\prime}}$. For simplicity, we shall refer to these two sets as " $A$ 's" and " $B$ 's". By reversing the orientation of some of the $B$ 's if necessary, we can assume they all have positive linking numbers with $A_{1}$. Now, $A_{2}$ has linking numbers of the same sign with at least half of the $B$ 's. We keep these $B$ 's and discard the rest. By reversing the orientation of $A_{2}$ if necessary, we can assume that $A_{2}$ has positive linking number with the $B$ 's that we kept. We repeat this process for $A_{3}, A_{4}, \ldots, A_{m_{n}}$, each time discarding at most half of the $B$ 's. This reduces the number of $B$ 's by a factor of at most $2^{m_{n}-1}$, leaving us with at least $m_{n}^{\prime} / 2^{m_{n}-1}=6 m_{n}+6$ remaining $B$ 's, which are each linked to all of the $A$ 's with linking number at least $\lambda$.

Next we apply Lemma 4 to the link whose components are $A_{1}, \ldots, A_{m_{n}}$ together with $6 m_{n}+6$ of the remaining $B$ 's. This gives us an oriented knot $Q_{1}$ which is linked to all the $A$ 's with absolute value of its linking number at 
least $\lambda$ and $\left|a_{2}\left(Q_{1}\right)\right| \geq \lambda^{2} / 16$. Let $L_{1}$ be the oriented link whose components are $A_{1}, \ldots, A_{m_{n}}$ together with $Q_{1}$. Then $L_{1}$ has $m_{n}+1=f^{n-1}(n)=m_{n-1}+$ $m_{n-1}^{\prime}$ components.

We can repeat the above process for the link $L_{1}$, beginning by partitioning the components of $L_{1}$ into a set of $m_{n-1}$ components which includes the knot $Q_{1}$ and a set of $m_{n-1}^{\prime}$ components. We abuse notation and refer to the first set as a set of $A$ 's and the second set as a set of $B$ 's. By applying the above argument to these sets of $A$ 's and $B$ 's we get an oriented link $L_{2}$ whose components are the $m_{n-1} A$ 's including $Q_{1}$, plus a new oriented knot $Q_{2}$ with $\left|a_{2}\left(Q_{2}\right)\right| \geq \lambda^{2} / 16$ such that $Q_{2}$ is linked to all of the $A$ 's with absolute value of its linking number at least $\lambda$.

We repeat the above process a total of $n-1$ times making sure that at each stage all of the $Q_{i}$ 's that we have constructed so far are included among the new $A$ 's. In this way we get the desired link with components $Q_{1}, \ldots, Q_{n}$, all of whose pairwise linking numbers have absolute value at least $\lambda$ and each component $Q_{i}$ satisfies $\left|a_{2}\left(Q_{i}\right)\right| \geq \lambda^{2} / 16$.

We now prove our main result using Lemma 5 and Theorem 1.

Theorem 2. For all $n, \alpha \in \mathbb{N}$, there is a complete graph $K_{r}$ such that every embedding of $K_{r}$ in $\mathbb{R}^{3}$ contains an oriented link with components $Q_{1}, \ldots, Q_{n}$ such that for every $i \neq j,\left|\operatorname{lk}\left(Q_{i}, Q_{j}\right)\right| \geq \alpha$ and $\left|a_{2}\left(Q_{i}\right)\right| \geq \alpha$.

Proof. Let $\lambda=\max \{\alpha, 4 \sqrt{\alpha}\}$. Let

$$
f(n)=n-1+(6 n) 2^{n-2} \text { and } m=f^{n}(n) .
$$

It follows from Theorem 1 that there is a complete graph $K_{r}$ such that every embedding of $K_{r}$ in $\mathbb{R}^{3}$ contains an oriented link $L_{0}$ with $m$ components such that the linking number of every pair of components of $L_{0}$ has absolute value at least $\lambda$. Now it follows from Lemma 5 that every embedding of $K_{r}$ in $\mathbb{R}^{3}$ contains an oriented link with components $Q_{1}, \ldots, Q_{n}$ such that, for any $i \neq j,\left|\operatorname{lk}\left(Q_{i}, Q_{j}\right)\right| \geq \lambda \geq \alpha$ and $\left|a_{2}\left(Q_{i}\right)\right| \geq \lambda^{2} / 16 \geq \alpha$.

Recall that it follows from Corollary 1 that there is no graph $G$ with the property that every embedding of $G$ in $\mathbb{R}^{3}$ contains a link with components $Q_{1}, \ldots, Q_{n}$ such that, for some orientation of the components, $\operatorname{lk}\left(Q_{i}, Q_{j}\right) \geq \lambda$ for any $i \neq j$. Thus the conclusion of Theorem 2 cannot be strengthened by removing the absolute value on the linking number.

\section{References}

[1] J. Conway and C. Gordon, Knots and links in spatial graphs, J. Graph Theory 7 (1983), 445-453. 
[2] E. Flapan, Intrinsic knotting and linking of complete graphs, Algebr. Geom. Topology 2 (2002), 371-380.

[3] E. Flapan, J. Foisy, R. Naimi and J. Pommersheim, Intrinsically n-linked graphs, J. Knot Theory Ramif. 10 (2001), 1143-1154.

[4] T. Fleming, Intrinsically linked graphs with knotted components, preprint: arXiv: 0705.2026v1 [math.GT].

[5] T. Fleming and A. Diesl, Intrinsically linked graphs and even linking number, Algebr. Geom. Topology 5 (2005) 1419-1432.

[6] F. P. Ramsey, On a problem of formal logic, Proc. London Math. Soc. (2) 30 (1930), 264-286.

[7] H. Sachs, On a spatial analogue of Kuratowski's theorem on planar graphs - an open problem, in: Graph Theory (Łagów, 1981), Lecture Notes in Math. 1018, Springer, Berlin, 1983, 230-241.

[8] —, On spatial representations of finite graphs, in: Finite and Infinite Sets, Vol. I, II (Eger, 1981), Colloq. Math. Soc. János Bolyai 37, North-Holland, Amsterdam, 1984, 649-662.

[9] K. Taniyama and A. Yasuhara, Realization of knots and links in a spatial graph, Topology Appl. 112 (2001), 87-109.

Department of Mathematics

Pomona College

Claremont, CA 91711, U.S.A.

E-mail: eflapan@pomona.edu

Department of Mathematics

Occidental College

Los Angeles, CA 90041, U.S.A.

E-mail: rnaimi@oxy.edu
Department of Mathematics Loyola Marymount University Los Angeles, CA 90045, U.S.A. E-mail: bmellor@lmu.edu

Received 4 December 2006;

in revised form 6 June 2008 